Results 8978 people in North East Essex recorded a register entry in the first five years of My Care Choices. The mean number of days to death from the initiation of a MCCR entry increased from 65 in year one, to 316 in year five. The proportion of people in North East Essex who died with a MCCR record increased from $20 \%$ in year one to $46 \%$ in year five. Preferred places of care were recorded for $81 \%$ of register entries. Of these $52 \%$ were home, $36 \%$ care home, $8 \%$ hospice, 3.5\% hospital, $0.5 \%$ community hospital. Achievement of first choice preferred place of care was $70 \%$.

Conclusion EPaCCs have potential to promote care consistent with peoples' preferences towards the end of life. Opportunities should be taken to develop systematic evaluations to drive improvement.

\section{P-96 MY CARE CHOICES REGISTER - BUILDING AN EPACCS FOR THE FUTURE}

Tim Clifton. St Helena, Colchester, UK

10.1136/bmjspcare-2019-HUKNC.119

Background In 2013, St Helena was commissioned to implement an EPaCCS (National End of Life Care Intelligence Network, 2013) solution across North East Essex that would be used to capture the choices of patients deemed to be in the last 12 months of life. These would be made available at point of care.

St Helena launched the My Care Choices Register (MCCR). It quickly became nested within the locality. However, the system procured had limitations and following stakeholder consultation it was decided that a bespoke solution was required.

Aims

- Reduce administrative overheads;

- Create a solution that provides value for money, has scope for future development and integrates with GPSoC;

- Ensure this system can be expanded for use across the healthcare system and aligns with LDR Universal Capabilities.

Method March 2017- after engaging stakeholders, St Helena created a specification for a new solution. Care IS, a clinical solutions developer with a large user base in North East Essex, was approached. Care IS agreed to develop the solution and offer this to other localities in the UK to aid commercial viability. September 2018 - New integrated MCCR is launched.

Results Record duplication issues have been eliminated. The replacement system is now utilised by more health care professionals and areas such as the Ambulance Service. The increased usage has led to a larger number of people dying in their preferred place of care.

Conclusion The integrated nature of MCCR and the increased usage have made it one of the success stories for North East Essex CCG. This is only the start. Phase Two will see MCCR expanded into social care, along with other localities currently in the planning stages to use MCCR across their footprint.

\section{P-97} DIGITAL CHANGE PROJECT: THE USE OF COORDINATE MY CARE (CMC) AT NORTH LONDON HOSPICE

Samantha Edward. North London Hospice, London, UK

\subsection{6/bmjspcare-2019-HUKNC.120}

Background Shared care records is identified as one of the foundations to achieving the Ambitions for Palliative and End of Life Care (National Palliative and End of Life Care Partnership, 2015). Electronic Palliative Care Co-ordination Systems (EPaCCS) are one vehicle which enable the recording and sharing of people's care preferences and key details about their care at the end of life. In North Central London (NCL) Coordinate My Care (CMC) has been adopted as the EPaCCs used across services and providers. Since January 2019 the community teams in the Boroughs of Barnet, Enfield and Haringey have undertaken a digital change project to improve use of CMC.

Objective To identify the challenges and enablers to achieving digital change.

Method Activity data provided by CMC was reviewed and evaluation of the ways in which digital change had been achieved is presented.

Results Since January 2019 for the Borough of Barnet the records created have more than tripled compared with the same time in 2018. Key themes in digital change management were explored according to the model suggested by the King's Fund (Maguire, Evans, Honeyman \& Omojomolo, 2018).

Leadership and management: strategic leadership:

- Commissioning- LPoL (Last Phase of Life) Programme;

- Key Performance Indicator;

- Objective setting: Teams and individual;

- Project management;

- Barriers or enablers for change identified;

- Clinical Leaders identified;

- Data - close the loop - identify individual champions and individual support needs.

Resourcing and skills:

- Financial investment;

- Technical access and support;

- Working with CMC access issues.

Information governance:

- Information Governance Framework;

- Consent - Information leaflets;

- Communication skills around data sharing and benefits;

- New starters, transfers and leavers;

- Legitimate access;

- Auditing.

User engagement:

- Clinical champions;

- Celebration of successes - Data;

- Considering less successful outcomes - what can be improved (multi-agency);

- Incentivisation;

- Whole team - multidisciplinary team, colleagues;

- Patient engagement - MyCMC (My Coordinate My Care).

Partnerships:

- Urgent care system: London Ambulance Service, GP Out of Hours, local Palliative Care Support Service and acute sector;

- Patient engagement - MyCMC. 